\title{
INTERSTELLAR DUST: OBSERVATIONS IN THE ULTRAVIOLET AND THEIR INTERPRETATIONS
}

\author{
A DOLF N. WITT
}

Ritter Astrophysical Research Center, The University of Toledo, U.S.A.

\begin{abstract}
Recent observations of interstellar extinction and diffuse galactic light in the ultraviolet are interpreted in terms of a bi-modal size distribution of interstellar grains.
\end{abstract}

The successful operation of the Orbiting Astronomical Observatory OAO-2 has provided abundant ultraviolet observations from which detailed information on the extinction and scattering properties of interstellar dust grains has been derived. Figure 1 summarizes some of these results. In the upper part the average wavelength dependence of interstellar extinction as determined by Bless and Savage (1972) is shown. In the lower part-using the same inverse wavelength scale-we plot the wavelength dependence of the albedo of the interstellar grains as determined by Witt and Lillie (1973) from an analysis of the diffuse galactic light observed in approximately 30 of Kapteyn's selected areas.

There is good evidence from the diffuse galactic light observations that the scattering at visible wavelengths is governed by a much more strongly forward-directed

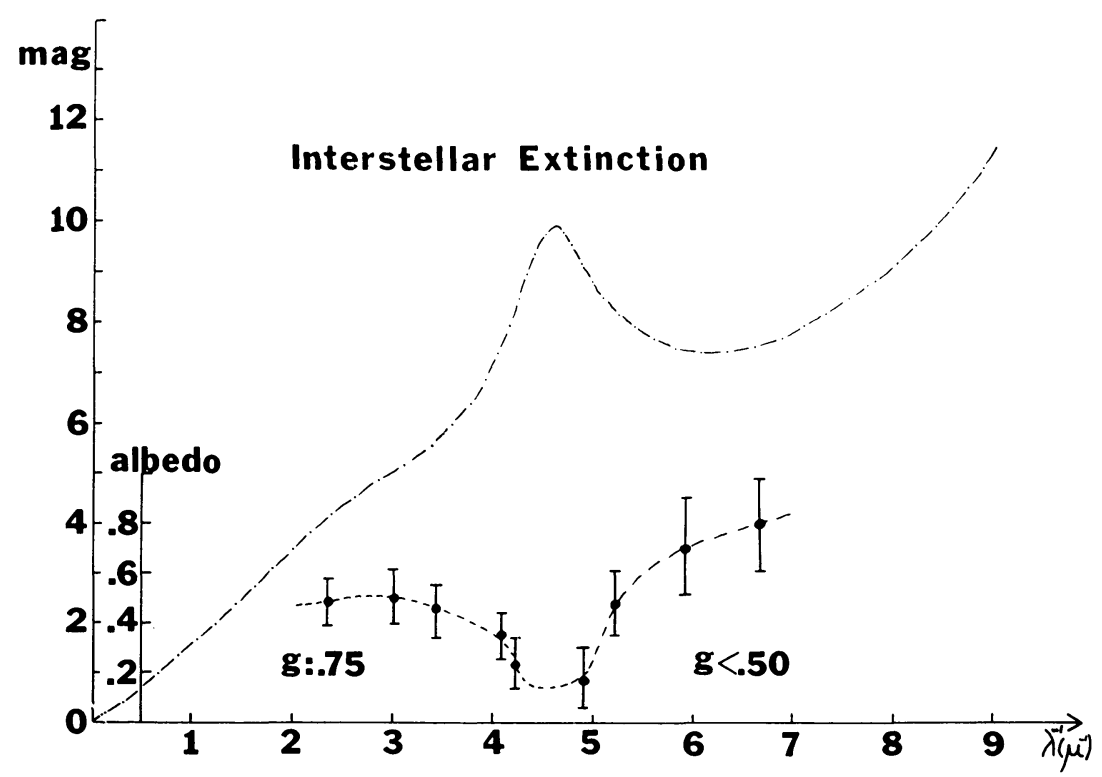

Fig. 1. The wavelength dependence of interstellar extinction as found by Bless and Savage (1972) and the wavelength dependence of the albedo of interstellar grains as determined by Witt and Lillie (1973). 
phase function as compared to the far-ultraviolet region, where the scattering appears to be more nearly isotropic. This result is indicated by the estimated values of the phase-function asymmetry of $g=0.75$ at around $4000 \AA$ wavelength and $g<0.5$ at wavelengths below $2000 \AA$.

The pronounced decrease of the albedo near $2200 \AA$ wavelength supports the idea of an absorption nature of the corresponding bump in the extinction curve. The subsequent rapid rise of the albedo shortward of $2000 \AA$ wavelength indicates that the far-ultraviolet extinction is caused by a scattering process. These results do not support the suggestion of Stecher and Williams (1969) that the far-ultraviolet rise in the extinction might be due to photo-dissociation of the $\mathrm{H}_{2}{ }^{+}$molecule.

Witt and Lillie (1973) have also derived the relative efficiencies for scattering and absorption of interstellar grains on the basis of the results shown in Figure 1. In Figure 2 these efficiencies are presented in comparison with the extinction curve.

How can these results be interpreted? It has been concluded by several authors in the past that the high ultraviolet extinction indicates the existence of a much larger proportion of small particles in interstellar space. Based on the result that the asymmetry factor of the scattering phase-function is considerably smaller in the far-ultraviolet than in the visible, a conclusion may be suggested that goes somewhat farther.

It appears quite impossible to construct a simple continuous size distribution of Mie-scattering particles of any given composition or index of refraction such that the scattering should become less forward - directed as one goes to shorter wavelengths, while it would still approximately reproduce the extinction curve. Therefore, it is suggested here that these data may support the existence of a bi-modal size distri-

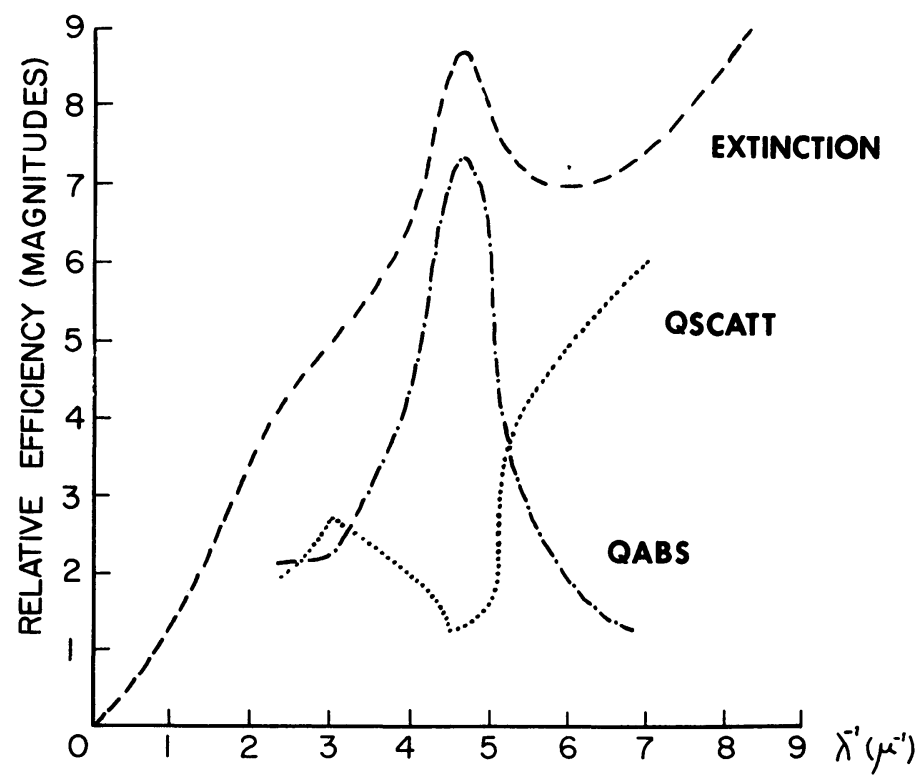

Fig. 2. The relative efficiencies for extinction, absorption, and scattering of interstellar grains. 
bution of interstellar dust particles. This distribution would contain large particles with diameters in the range of $5000 \AA$ and larger, and small particles with diameters of $500 \AA$ and smaller. The large particles would provide extinction in the visible region including scattering which is strongly forward directed, whereas the small particles needed in large numbers due to their small intrinsic efficiencies - would dominate the ultraviolet region and contribute nearly isotropic scattering. It would also be very reasonable to assume that particles existing in these two size ranges would be chemically different and would have resulted from quite different processes of formation or sources of origin.

A very simple model along these lines has been examined in order to study the conditions under which the observed properties of interstellar grains may be approximated. Mie calculations for the following ingredients were carried out: large, icy particles with a diameter of $5000 \AA$ and an index of refraction $n=1.33-0.1 i$, small graphite particles with a diameter of $400 \AA$, and small dielectric particles with a diameter of $400 \AA$ and $n=1.66$. There are only two variable parameters in the model: the respective ratios of the number of graphite particles or dielectric particles to the number of large icy particles.

While the chemical composition selected for the grains is in no way original, the size spectrum proposed here appears to be required by the scattered light data. The fact that small particles of two compositions are suggested is based on the observations by Bless and Savage (1972) that the magnitude of the absorption bump at $2200 \AA$ appears to be quite unrelated to the amount of far-ultraviolet extinction. The bump is thought to be due to graphite grains in interstellar space.

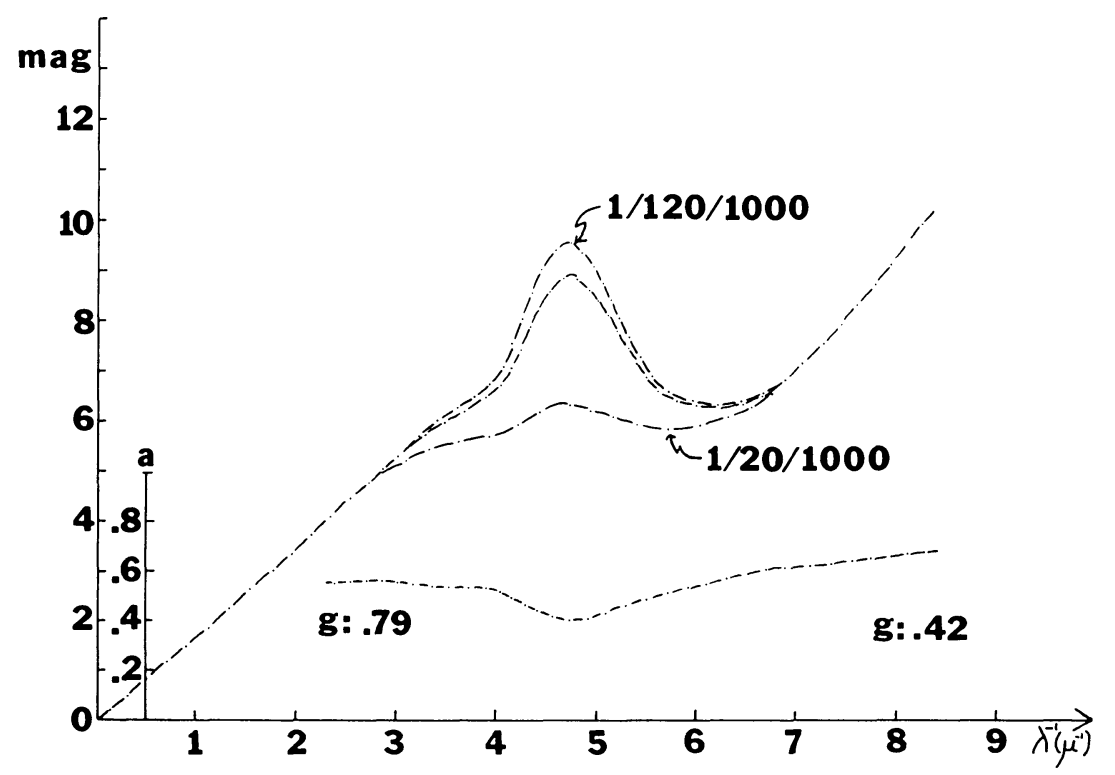

Fig. 3. Theoretical extinction and albedo curves for a bi-modal size distribution of interstellar grains. The effect of varying the relative number of graphite particles is shown. 
What number ratios of particles do we require in order to produce the observed extinction curve and how does the variation of these ratios affect the appearance of the resulting wavelength dependence of extinction. Figure 3 indicates that a mixture of one large particle to every 100 small graphite grains and every 1000 small dielectric grains appears to yield an extinction curve closely resembling the average observed one. The variation of the number of the graphite grains alone changes the magnitude of the absorption peak at $\lambda 2200$ without affecting the remainder of the curve. In the lower part of Figure 3 the theoretical albedo curve is plotted for this model. Qualitatively, it has the observed features with a minimum at $2200 \AA$ wavelength and a rise in the ultraviolet, but, quantitatively, the agreement with observations is not perfect. Better data on the indices of refraction of different materials are obviously needed for the ultraviolet, in particular, the question of the existence of materials which remain dielectric into the far-ultraviolet requires further study.

The principal result of the bi-modal size distribution is that the phase-function of scattering, in agreement with the observations, is strongly forward directed in the visible and tends to be more isotropic in the far-ultraviolet, as indicated by the values of the asymmetry factor $g=0.79$ and $g=0.42$, respectively.

Figure 4 illustrates the effect of varying the relative number of small dielectric particles in the size distribution. The amount of far-ultraviolet extinction varies accordingly, without producing much change in the other parts of the spectrum. Also, in accordance with the observations of Bless and Savage (1972), the position of the far-ultraviolet extinction minimum moves to shorter and shorter wavelengths as the

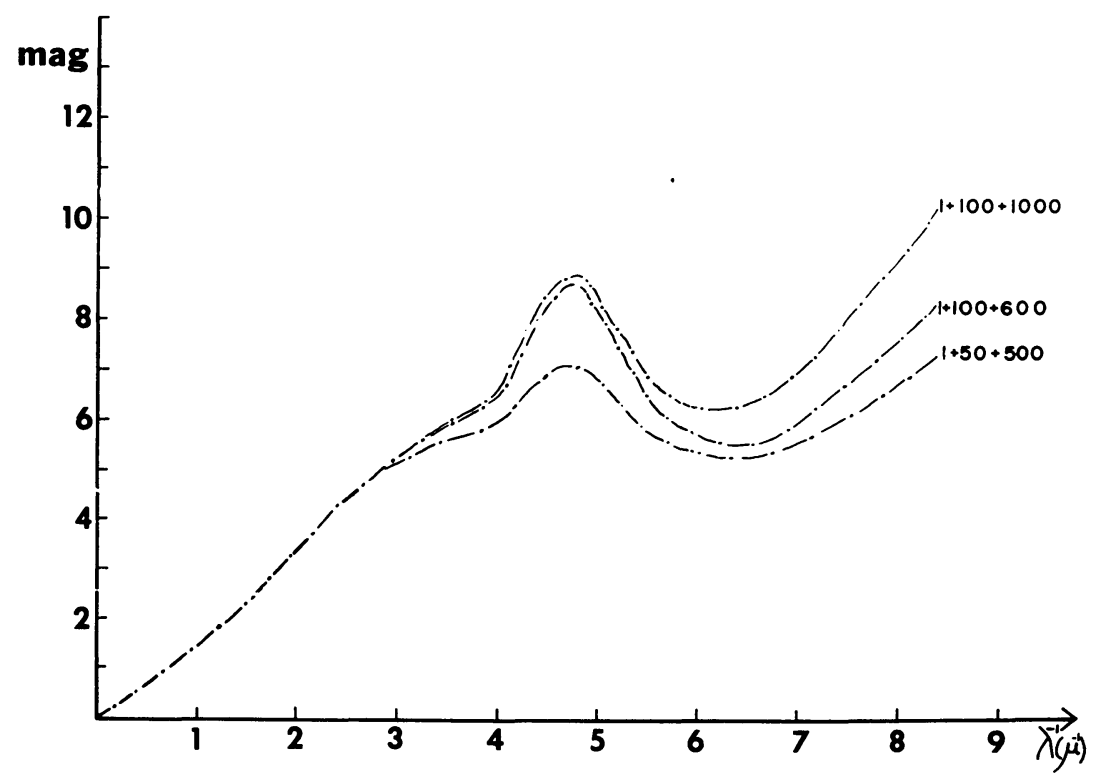

Fig. 4. Theoretical extinction curves for a bi-modal size distribution of interstellar grains. The effects of changing the contribution of small dielectric particles as well as all small particles is demonstrated. 
extinction in the extreme UV diminishes. Finally, when both small particle components are reduced in number, the extinction can become nearly grey in the UV, which is similar to a case observed in Orion.

In summary, as a result of observations of the diffuse galactic light the existence of a bi-modal size distribution of interstellar dust particles is suggested. It has been shown that a very simple model which incorporates particles of the two basic size ranges can indeed explain the observations of interstellar extinction and diffuse galactic light.

\section{References}

Bless, R. C. and Savage, B. D.: 1972, Astrophys. J. 171, 293.

Stecher, T. P. and Williams, D. A.: 1969, Astrophys. Letters 4, 99.

Witt, A. N. and Lillie, C. F.: 1973, Astron. Astrophys. 25, 397. 